\title{
PD-L1 Expression Promotes Epithelial to Mesenchymal Transition in Human Esophageal Cancer
}

\author{
Lujun Chen ${ }^{a, b, c, d} \quad$ Yuqi Xiong ${ }^{a, b, c}$ Jing Lia,b,c Xiao Zheng ${ }^{a, b, c} \quad$ Qi Zhou ${ }^{a, b, c}$ \\ Abbey Turnerd $^{d}$ Changping Wu $u^{a, b, c}$ Binfeng Lu ${ }^{d}$ Jingting Jiang ${ }^{a, b, c}$
}

aDepartment of Tumor Biological Treatment, bjiangsu Engineering Research Center for Tumor Immunotherapy and 'Institute of Cell Therapy, the Third Affiliated Hospital of Soochow University, Jiangsu Changzhou, China; 'Department of Immunology, University of Pittsburgh, Pittsburgh, PA, USA

\section{Key Words}

PD-L1 • Epithelial to mesenchymal transition • Esophageal cancer

\begin{abstract}
Background/Aims: PD-L1 (Programmed cell death 1 ligand 1, PD-L1), an essential immune checkpoint molecule in the tumor microenvironment, is an important target for cancer immunotherapy. We have previously reported that its expression in human gastric and esophageal cancer tissues is significantly associated with cancer progression and patients' postoperative prognoses. Its expression in cancer cells is well known to inhibit the T cellmediated anti-tumor response, and this mechanism of action has been targeted for cancer immunotherapy. As of now, the autonomous effect of PD-L1 on cancer cells is not well understood, thus our present study aimed to examine the role of PD-L1 intervention in cellular biological functions, especially epithelial to mesenchymal transition (EMT), of the human esophageal cancer cell line, Eca-109 cells. Methods: Immunohistochemistry assay was used to investigate the correlation between expression of PD-L1 and EMT markers in human esophageal cancer tissues. Intervention of PD-L1 by using RNAi and over-expression methods were used to study the role of PD-L1 in regulation of biological behaviors and EMT in Eca109 cells. Results: Our clinical and pathological data demonstrated that tumor samples in the EMT positive subgroup had higher PD-L1 expression than those in the EMT negative subgroup. By manipulating PD-L1 expression in Eca-109 cells either through ablation or overexpression of wild type and the cytoplasmic domain-truncated mutant, we demonstrated that PD-L1 expression significantly promoted the cell viability, migration and EMT phenotype. Furthermore, our study also indicated that PD-1 fusion protein mediated stimulation of PD-L1 and the cytoplasmic domain of PD-L1 played a critical role in promoting EMT phenotype of Eca-109 cells, thereby suggesting that PD-1 receptor usually by triggering the reverse signaling can effect PD-L1 mediated regulation of esophageal cancer cell response. Conclusion: Our present study reveals a tumor cell-autonomous role of PD-L1 signaling in promoting EMT in human esophageal cancer.

L. Chen and Y. Xiong contributed equally to this work.




\section{Introduction}

PD-L1, also known as B7-H1 or CD274, is an essential immunomodulatory ligand from the B7/CD28 family. It negatively regulates $\mathrm{T}$ cell activation and effector function by interacting with its receptor, PD-1, expressed on T cells. Thus, it plays an important role in dampening the $T$ cell mediated anti-tumor immune response $[1,2]$. Numerous studies have shown that many human cancers display PD-L1 over-expression, and that its expression is significantly associated with patients' clinical parameters and postoperative prognoses [3-5]. In addition, multiple clinical studies have demonstrated that PD-L1 blockade can significantly inhibit tumor progression and improve patient prognosis in many advanced cancers, including melanoma, gastric cancer, non-small-cell lung cancer, ovarian cancer, renal cell cancer and so on. Thus, these studies have highlighted that anti-PD-L1 therapy holds great promise for the treatment of human malignancies $[6,7]$.

Outside of the negative regulation associated with PD-L1 expression in the T cellmediated anti-tumor response, the contribution of PD-L1 expression in regulating the biological behaviors of the tumor cells themselves, such as proliferation, apoptosis, migration and resistance to chemo/radio-therapy, is still not clearly understood [8-14]. Our recent study has shown that knockdown of PD-L1 expression in the human gastric cancer cell line, SGC-7901, could significantly suppress cell proliferation, migration and tumor growth in the subcutaneous transplantation mouse model [15]. Similarly, in human colorectal cancer, the study by Shi et al. demonstrated that positive PD-L1 expression is an independent predictor for cancer patients' prognoses, and its knockdown can inhibit cell proliferation and migration [16].

Interestingly, there is also compelling evidence concerning the role of PD-L1 in promoting epithelial to mesenchymal transition (EMT) as well as initiation and maintenance of cancer stem cells [17-21]. For instance, a study carried out by Alsuliman et al. indicated that induction of epithelial to mesenchymal transition in human mammary epithelial cells up-regulated PD-L1 expression mainly through activation of the PI3K/AKT pathway [10]. In another study, it was shown that PD-L1 expression could induce epithelial-to-mesenchymal transition in renal cell carcinoma by activating the transcription factor-SREBP-1c [22]. Notably, the survival analysis in the TCGA database confirmed that prognoses were better for PD-L1 ${ }^{+} /$EMT $^{-}$patients than for PD-L1 ${ }^{+} / \mathrm{EMT}^{+}$patients with human head and neck squamous cell carcinoma [23]. In our previously published studies, we have separately identified PD-L1 expression and EMT as important prognostic factors in human esophageal cancer [24, 25]. Nevertheless, the correlation between the abnormal expression of PD-L1 and EMT status in the progression and metastasis of human esophageal cancer still remains elusive.

In the current study, we have specifically focused on establishing the correlation between PD-L1 expression and EMT status in human esophageal cancer tissues, and further investigated the role of PD-L1 in regulating the EMT phenotype in the human esophageal cancer cell line, Eca-109. Our immunohistochemistry results indicated that higher PD-L1 expression was correlated with positive EMT status in contrast to negative EMT status. Using various cellular models including PD-L1 knockdown and overexpression of wide type as well as cytoplasmic domain truncation of PD-L1 in human esophageal cancer cell line Eca-109, we demonstrated that intervention of PD-L1 expression could significantly alter biological behaviors including cell viability and EMT phenotype. In addition, by PD-1 fusion protein stimulation, we also showed that the cytoplasmic domain of PD-L1 plays a critical role in regulating the EMT phenotype of Eca-109 cells.

\section{Materials and Methods}

Patients and tissue samples

Formalin-fixed, paraffin-embedded esophageal cancer tissue samples were collected from 99 patients who underwent surgical resection between February 2005 and May 2006 in the Third Affiliated Hospital 


\section{Cellular Physiology Cell Physiol Biochem 2017;42:2267-2280

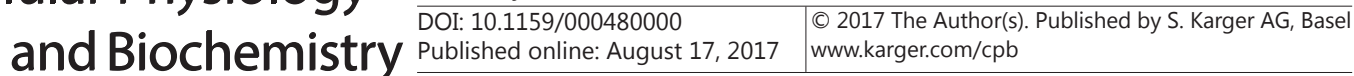 \\ Published onlıne: August 17, 2017 www.karger.com/cp}

of Soochow University ( 76 men and 23 women; median age at diagnosis, 59 years). None of these patients received pre-operative chemotherapy or radiotherapy, and all tumor tissues were confirmed as esophageal squamous cell carcinomas by using hematoxylin and eosin (H\&E) staining after surgical resection. Survival data was available for 56 patients, and was used for survival analysis.

\section{Antibodies and other reagents}

Rabbit anti-human PD-L1 monoclonal antibody (NBP1-03220) used for the immunohistochemistry assay was purchased from Novus Biologicals (Littleton, CO, USA). APC-conjugated, mouse anti-human PDL1 and mouse IgG1 isotype control monoclonal antibodies were purchased from BioLegend (San Diego, CA, USA). Mouse anti-human E-cadherin (MAB-0589) and Vimentin (MAB-0178) monoclonal antibodies used for the immunohistochemistry assay were purchased from Maixin Biotechnology (Fuzhou, China). The HRP-labeled goat anti mouse/rabbit secondary antibodies (K500711) were purchased from Dako (Glostrup, Denmark). In addition, rabbit anti-human GAPDH (Sigma, St. Louis, MO, USA), rabbit anti-human PD-L1 and rabbit anti human $\beta$-actin (Abcam, Cambridge, MA, USA), mouse anti-human E-cadherin (Cell Signaling, Danvers, MA, USA), rabbit anti-human N-Cadherin and rabbit anti-human Zeb1 (Santa Cruz, Dallas, TX, USA), goat anti-mouse IgG and goat anti-rabbit IgG (Sigma, St. Louis, MO, USA) were used for western blot analysis. The RNeasy Mini Kit was purchased from Qiagen (Valencia, CA, USA), and SYBR Green Master Mix kits were purchased from TaKaRa (Dalian, China). RPMI-1640 media and fetal bovine serum (FBS) were purchased from Gibco (Cambrex, MD, USA).

\section{Immunohistochemical staining and analysis}

The immunostaining of PD-L1, E-cadherin and vimentin proteins in human esophageal cancer tissues was performed according to our previously published protocol [24, 25]. First, the paraffin-embedded tumor tissue blocks were cut into serial 3- $\mu \mathrm{m}$-thick sections, dewaxed using xylene, and rehydrated and graded in ethanol solutions. Next, antigen retrieval was performed by heating the tissue sections at $100^{\circ} \mathrm{C}$ for 30 min in a citrate solution $(10 \mathrm{mmol} / \mathrm{L}, \mathrm{PH} 6.0)$, and after cooling of the sections, they were immersed in $0.3 \%$ hydrogen peroxide solution for $15 \mathrm{~min}$ to block endogenous peroxidase activity. This was followed by rinsing sections in PBS for 5 min and blocking with 3\% BSA solution at room temperature for 20 min. Subsequently, the sections were incubated with rabbit anti-human PD-L1 polyclonal antibody, mouse anti-human E-cadherin monoclonal antibody, and mouse anti-human Vimentin monoclonal antibody, respectively, at $4^{\circ} \mathrm{C}$ overnight. The next day, the sections were incubated with HRP-labeled goat anti mouse/ rabbit secondary antibody, and diaminobenzene was used as the chromogen and hematoxylin as the nuclear counterstain. Finally, the sections were dehydrated, cleared and mounted.

All slides were examined independently by two senior pathologists who were blind to the patients' clinical parameters. The immunostaining intensities of PD-L1 and E-cadherin were assessed according to the $H$-score method, as described previously [26, 27]: $H$-score $=(\%$ tumor cells unstained $\mathrm{x} 0)+(\%$ tumor cells stained weak $\mathrm{x} 1)+(\%$ tumor cells stained moderate $\mathrm{x} 2)+(\%$ tumor cells stained strong $\mathrm{x} 3)$. The $H$-scores ranged from 0 (100\% negative tumor cells) to 300 (100\% strong staining tumor cells). To evaluate vimentin staining, the ratio of vimentin positive cells within all cancer cells was calculated and the positive rate was recorded. The scoring results from the two pathologists for each protein were averaged and used for statistical analysis.

\section{Cell culture}

The human esophageal cancer cell line, Eca-109 was purchased from Chinese Academy of Sciences, Shanghai Institutes for Biological Sciences (Shanghai, China), and was cultured in RPMI1640 media, and was supplemented with $10 \% \mathrm{FBS}$ in the presence of benzylpenicillin $(100 \mathrm{U} / \mathrm{ml})$, streptomycin $(100 \mu \mathrm{g} /$ $\mathrm{ml})$ and $2 \mathrm{mM} \mathrm{L-glutamine}$. The cells were incubated under standard-culture conditions $\left(5 \% \mathrm{CO}_{2}, 37^{\circ} \mathrm{C}\right)$.

\section{RNAi lentivirus generation, infection and cell sorting}

The human esophageal cancer cell line Eca-109 was used for the knockdown expression of PD-L1. The small hairpin RNA (shRNA) against the human PD-L1 gene (NM_014143.2; GenBank) was obtained from Shanghai GeneChem Co. Ltd (Shanghai, China), and cloned into a lentiviral gene transfer vector encoding green fluorescent protein (GFP). The two following shRNA target sequences against PD-L1 were used. shRNA-1: 5'-GACCTATATGTGGTAGAGTAT-3', and shRNA-2: 5'-CGAATTACTGTGAAAGTCAAT-3'. The 


\section{Cellular Physiology Cell Physiol Biochem 2017;42:2267-2280

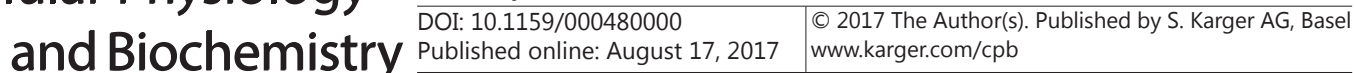 \\ Chen et al.: PD-L1 Promotes EMT in Esophageal Cancer}

recombinant PD-L1 targeting lentivirus (LV-PD-L1-shRNA virus) and control mock lentivirus (LV-NC virus) were prepared and transfected in to Eca-109 cells. Later, the infected cells were analyzed by flow cytometry (Canto II, BD, USA). Positive cells were sorted using a GFP tag in an Aria II flow sorter (BD Bioscience, NJ, USA). The positive sorted cells were then named LV-PD-L1-shRNA-1, LV-PD-L1-shRNA-2, and LV-NC.

Construction of PD-L1 over-expression in Eca-109 cells

To generate full-length and truncated PD-L1, the gene fragments were synthesized by Sangon Biotech Shanghai Co., Ltd. (Shanghai, China) and amplified by PCR. The primers were designed as follows: Forward primer of full length PD-L1: 5'-TAGAATTCATGAGGATATTTGCTGTCTT-3'; reverse primer of full length PDL1: 5'-TAGGATCCTTACGTCTCCTCCAAATGTG-3'; forward primer of cytoplasmic domain truncated PD-L1: 5'-TAGAATTCATGAGGATATTTGCTGTCTT-3'; reverse primer of cytoplasmic domain truncated PD-L1: 5'-TAGGATCCTTATTTTCTTAAACGGAAGA-3'.The PCR fragment was subcloned into the EcoRI and BamHI sites in the pLV-IRES-ZsGreen-T2A-puro vector (Promega Biotech Co., Ltd., Madison, WI, USA).

Then the Eca-109 cells were transfected with the recombinant lentivirus and sorted using a GFP tag. The positive sorted cells were then named LV-PD-L1-WT-OE (Eca-109 cells over-expressing wild type PDL1), LV-PD-L1-TN-OE (Eca-109 cells over-expressing cytoplasmic truncated PD-L1), and LV-Vector-Ctrl (Eca-109 cells over-expressing vector control).

\section{Real-time polymerase chain reaction (RT-PCR)}

The mRNA expression of PD-L1 in different cellular models of Eca-109 cell line was quantified by RT-PCR. Total RNA from the cell lines was extracted by using TRIzol (Invitrogen) method, and PCR reactions were performed using the ABI 7600 system (Applied Biosystems, USA) according to the manufacturer's instructions. Primer sequences for the detection of the reference gene (GAPDH) and the target gene (PD-L1) were synthesized as follows: human GAPDH forward primer: 5'-TGACTTCAACAGCGACACCCA-3', human GAPDH reverse primer: 5'-CACCCTGTTGCTGTAGCCAAA-3', intracellular fragment of human PD-L1 forward primer: 5'- TACCTCTGGCACATCCTCCA-3', intracellular fragment of PD-L1 reverse primer: 5'-ACGTCTCCTCCAAATGTGTATCA-3', extracellular fragment of human PD-L1 forward primer: 5'-ACTGGCATTTGCTGAACG-3', and extracellular fragment of PD-L1 reverse primer: 5'-TCCTCCATTTCCCAATAGAC-3'. Data was analyzed by comparing expression using Ct (2- $\Delta \Delta \mathrm{Ct}$ ) method, and values were expressed as fold change compared to GAPDH expression. The RT-PCR products for GAPDH and PD-L1 were also confirmed by electrophoresis using a $1.8 \%$ agarose gel containing $0.1 \%$ ethidium bromide.

\section{Western blot analysis}

The levels of PD-L1, E-cadherin, N-cadherin, Zeb1, Vimentin and GAPDH proteins in different cellular models were determined by western blot analysis. In brief, whole cell extracts were prepared from $1 \times 10^{6}$ cells using RIPA lysis buffer (50 mMTris/HCl pH 7.4, $150 \mathrm{mMNaCl}, 1 \%$ Nonidet P-40, 0.25\% Na-eoxycholate, $1 \mathrm{mM}$ EDTA and protease inhibitor cocktail). Cells were lysed on ice for $30 \mathrm{~min}$, and the lysates were collected in microtubes and centrifuged for $15 \mathrm{~min}$ at $12000 \mathrm{rpm} / \mathrm{min}$ at $4^{\circ} \mathrm{C}$. After centrifugation, supernatants were collected and the protein concentrations were measured by using a BCA Protein Assay Kit (Beyotime, Jiangsu, China). Equal amounts of denatured proteins were separated by SDS-PAGE and transferred to PVDF membrane (Millipore). The membranes were later blocked by using $5 \%$ non-fat dry milk in TBS-T (20 mMTris, pH 7.4, $137 \mathrm{mMNaCl}, 0.05 \%$ Tween-20) for 3 hours at room temperature, and then were incubated with primary antibodies overnight at $4^{\circ} \mathrm{C}$. The next day, blots were washed and incubated for 1 hour with anti-rabbit or anti-mouse secondary antibodies and washed three times with PBST. Finally, immuno-reactive protein bands were detected using an Odyssey Scanning system (Li-Cor, Lincoln, NE, USA).

\section{Cell viability assay}

Cell viability was assessed by using Cell Counting Kit-8 (CCK-8, Beyotime, Shanghai, China), according to the manufacturer's instructions. The Eca-109 cells $\left(5 \times 10^{3}\right)$ from LV-NC, LV-PD-L1-shRNA-1, LV-PD-L1shRNA-2, LV-Vector-Ctrl, LV-PD-L1-TN-OE, and LV-PD-L1-WT-OE groups were seeded into 96-well plates and incubated for 24, 48, 72 and 96 hours. CCK-8 reagent was added to each well at 3 hours before the endpoint of incubation, and absorbance was measured at $450 \mathrm{~nm}$ for each well by a microplate reader. An increase or decrease in the absorbance values at $450 \mathrm{~nm}$ in the experimental wells relative to the initial 


\section{Cellular Physiology Cell Physiol Biochem 2017;42:2267-2280

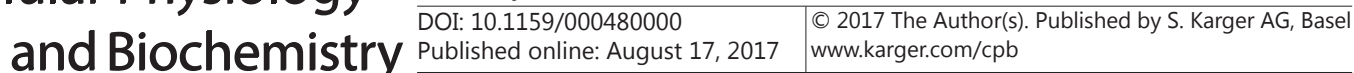 \\ Chen et al.: PD-L1 Promotes EMT in Esophageal Cancer}

value indicated cell growth or death, respectively. Each experiment was repeated at least three times in triplicate.

\section{Wound healing assay}

The role of PD-L1 in cell migration was evaluated by wound scrape assay. In brief, the different cellular models of Eca-109 cells were cultured in 6-well plates. A small wound area was created using a $200 \mu \mathrm{l}$ pipette tip when each cell type had grown to a $90 \%$ confluent monolayer. Cells were subsequently washed twice with PBS and then incubated in serum-free RPMI- 1640 media at $37^{\circ} \mathrm{C}$ in a $5 \% \mathrm{CO}_{2}$ incubator for $48 \mathrm{hrs}$. Photographs were taken at 3 different time points, 0,24 and 48 hours. Wound width was measured using a BX50 microscope (Olympus ${ }^{\circledR}$ ) with a calibrated eyepiece grid. Data from experiments done in triplicate was averaged and expressed as a percent of the original width.

\section{Transwell assay}

The co-culture system was also used to evaluate the influence of PD-L1 on the migration ability of Eca-109 cells, as described previously $[28,29]$. The transwell assay was performed using Matrigel-coated invasion chambers (Corning, NY, USA). The cells $\left(2 \times 10^{5}\right.$ cells/well) from the six different groups were serum starved in the upper chamber for 24 hours. Next, medium containing 10\% FBS was placed in the lower chamber as a chemo attractant. After 48 hours of incubation, cells that had migrated into the lower chamber were collected and resuspended, and non-migrating cells were removed from the top of the Matrigel with a cotton-tipped swab. Migrating cells were fixed and stained with $0.1 \%$ crystal violet and later photographed under a microscope (Olympus, Tokyo, Japan). Finally, the migrated cells were counted from five random fields.

Statistical analyses

Statistical analysis was completed using the paired Student's $t$-test, the Wilcoxon signed rank test, the Chi-square test or the Log-rank survival analysis where appropriate for final analysis of the data. The data are shown as the mean and range or mean \pm SD of three independent experiments. All the statistical analyses were performed using the GraphPad Prism 5.0 software package (GraphPad Software, Inc., San Diego, USA). A $p$-value of $<0.05$ represented significance.

\section{Results}

Correlation of PD-L1 and EMT markers (E-cadherin and vimentin) expression with human esophageal cancer patient's prognosis

In an effort to investigate the combined prognostic value of PD-L1 expression and EMT status in human esophageal cancer, we performed immunohistochemical staining of PD-L1 and EMT markers, E-cadherin and vimentin, in different human esophageal cancer tissues. As seen in Fig. 1A, immunostaining analysis of human esophageal cancer tissues showed that low/negative PD-L1 staining, positive E-cadherin staining and negative vimentin staining were identified in highly-differentiated esophageal cancer tissues. In contrast, positive PDL1 staining, negative E-cadherin staining and positive vimentin staining were observed in poorly-differentiated esophageal cancer tissues. Based on EMT marker staining, we categorized all 99 tissue sections from esophageal cancer patients into two major subgroups; 1) EMT positive subgroup $(n=60)$, including patients with E-cadherin negative/weak $(H$-score $<50)$ and vimentin positive (positive rate>0) staining, 2) EMT negative subgroup $(\mathrm{n}=39)$ which included all other patients. Among these two groups, we observed that PD-L1 staining intensity was significantly higher in the EMT positive subgroup-as compared to the EMT negative subgroup $(P=0.0314)$, as seen in Fig. 1B. Moreover, the survival analysis also indicated that the patients with lowPD-L1 (H-score $\leq 15)$ and EMT negative status had better overall survival than those with highPD-L1 expression and EMT positive status (HR=2.876, 95\%CI: 1.182 6.997, $P=0.0199$ ), as seen in Fig. 1C. 
Fig. 1. Analysis of PD-L1, E-cadherin and vimentin expression, and their correlation with esophageal cancer patient survival. A. Immunohistochemical staining analysis of PD-L1, E-cadherin and vimentin in highly (upper panel) and poorly (lower panel) differentiated human esophageal cancer tissues sections. Scale bar: $100 \mu \mathrm{m}$. B. Comparison of PD-L1 staining intensity among EMT positive and negative subgroup patients. C. Comparative overall survival analysis of patients with varying PD-L1 and EMT marker expression patterns.

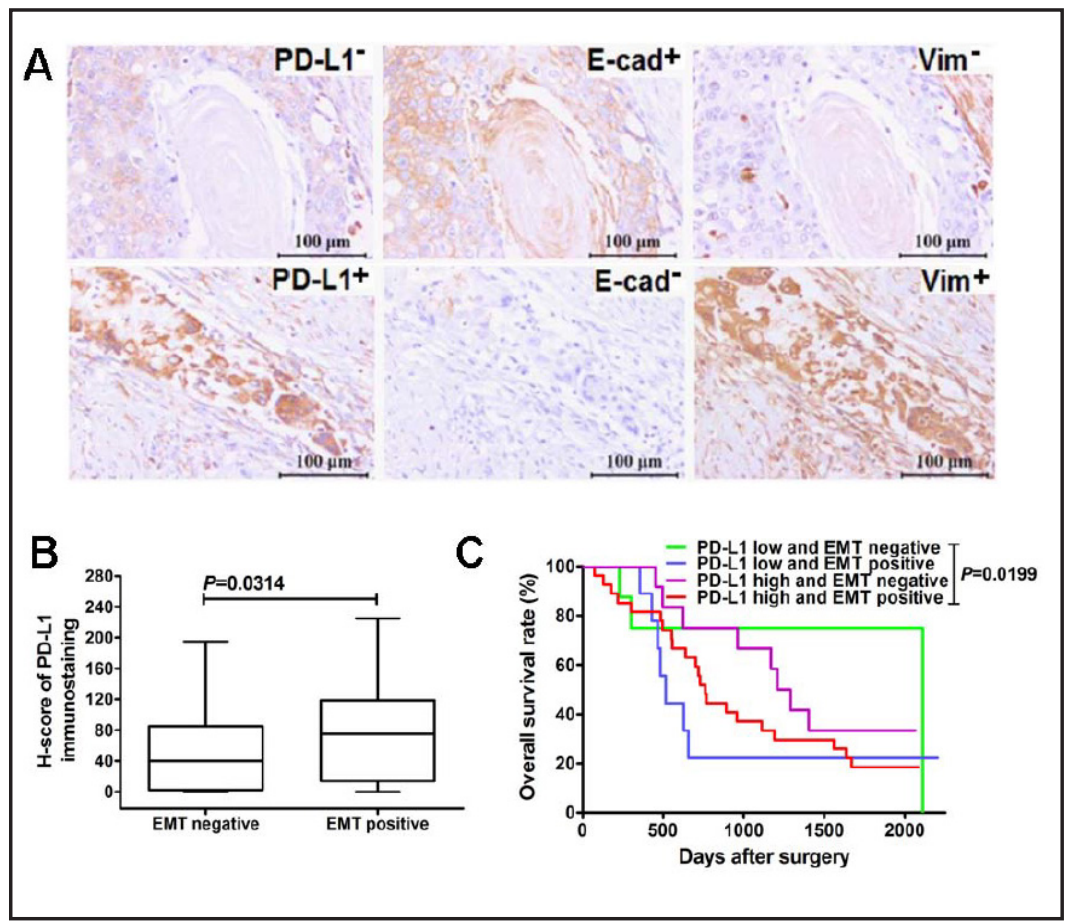

Fig. 2. GFP expression-based assessment of the lentivirus infection efficiency. The infection efficiency of different lenti-viruses expressing either PD-L1 shRNAs or over-expressing wild type and cytoplasmic truncated mutant, was confirmed in ECa-109 cells by detecting GFP expression via fluorescence microscopy. The top panel shows phase contrast images, while the lower panel shows images with GFP positive cells. Scale bar $=100 \mu \mathrm{m}$.

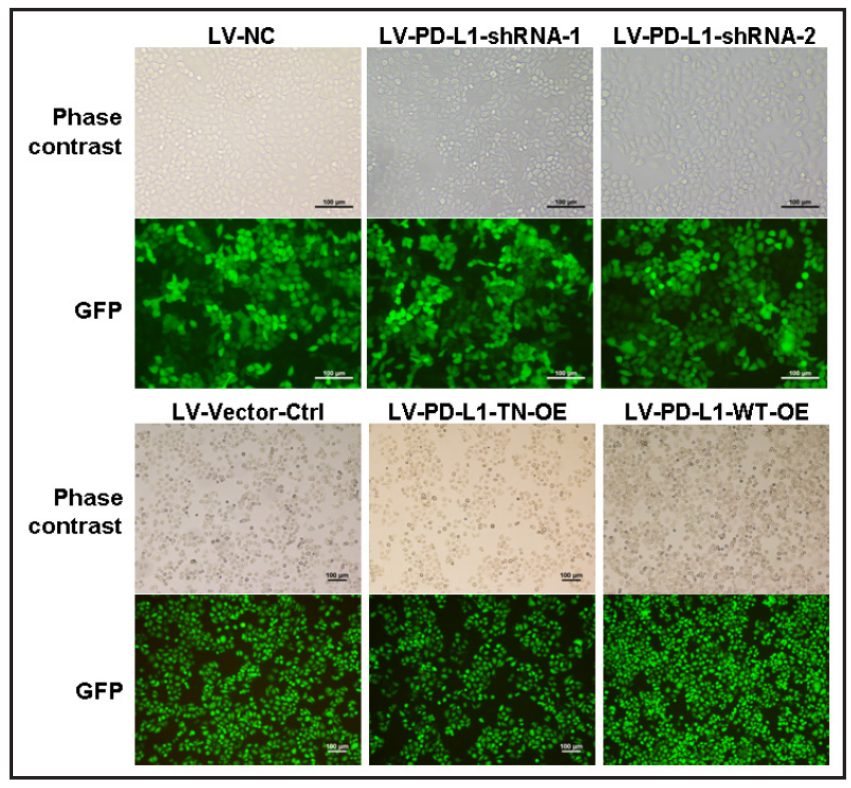

PD-L1 ablation and overexpression of wild type and cytoplasmic mutant in human esophageal cancer cell line Eca-109

To further investigate the role of PD-L1 in regulating the biological features of human esophageal cancer cells, we manipulated its expression in human esophageal cancer cell line, Eca-109. We stably ablated PD-L1 expression using two different shRNAs by lentivirus mediated infection of Eca-109 cells, (LV-PD-L1-shRNA-1 and LV-PD-L1-shRNA-2). The efficiency of infection was confirmed by detecting GFP expression via fluorescence microscopy (Fig. 2). We also over-expressed wild type PD-L1 (LV-PD-L1-WT-OE) and PDL1 truncated for cytoplasmic fragment (LV-PD-L1-TN-OE). The expression of PD-L1 was confirmed by both PCR methods in different cellular models. As seen in Fig. 3A, the mRNA expression level of the intracellular fragment of PD-L1 was reduced in both LV-PD-L1shRNA-1 and LV-PD-L1-shRNA-2 cells in contrast to LC-NC cells, while it was increased in 
Fig. 3. The mRNA as well as protein expression analysis of PD-L1 in Eca-109 cells following both its ablation and overexpression. A. Agarose gel displaying mRNA expression of intracellular (upper row) and extra-cellular fragments (middle row) of PD-L1 in Eca-109 cells after ablation with two different shRNAs (LV-PD-L1-shRNA-1 and LV-PD-L1-shRNA-2), and overexpression of wild type (LV-PD-L1-WT-OE) and cytoplasmic truncated mutant (LV-PD-L1-TN-OE). Bottom row shows GAPDH mRNA expression. LV-NC indicates mock lentiviral infection and LV-vector-Ctrl indicates control vector transfection. B. mRNA expression of the intracellular fragment of PD-L1 normalized to GAPDH expression. C. mRNA expression of the extracellular fragment of PD-L1 normalized to GAPDH expression. D. Protein expression of PDL1, as analyzed by western blot (bottom row) in different cellular models of Eca109 cells after stable ablation or overexpression. The upper row shows $\beta$-actin protein expression as a control. E. Relative protein expression of PD-L1 after normalization with $\beta$-actin expression. F. Flow cytometric analysis of PD-L1 expression in different cellular models of Eca109 cells. "*” represents a $P$ value of $<0.05$, “**” represents a $P$ value of $<0.01$, and “***" represents a $P$ value of $<0.001$. N.S. represents non-significant.
A

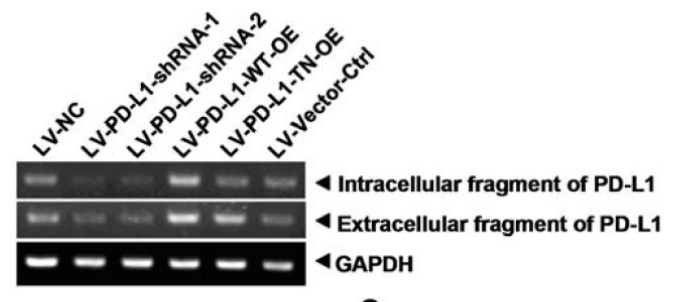

B C Extracellular fragment of $\mathrm{PD}-\mathrm{L} 1$
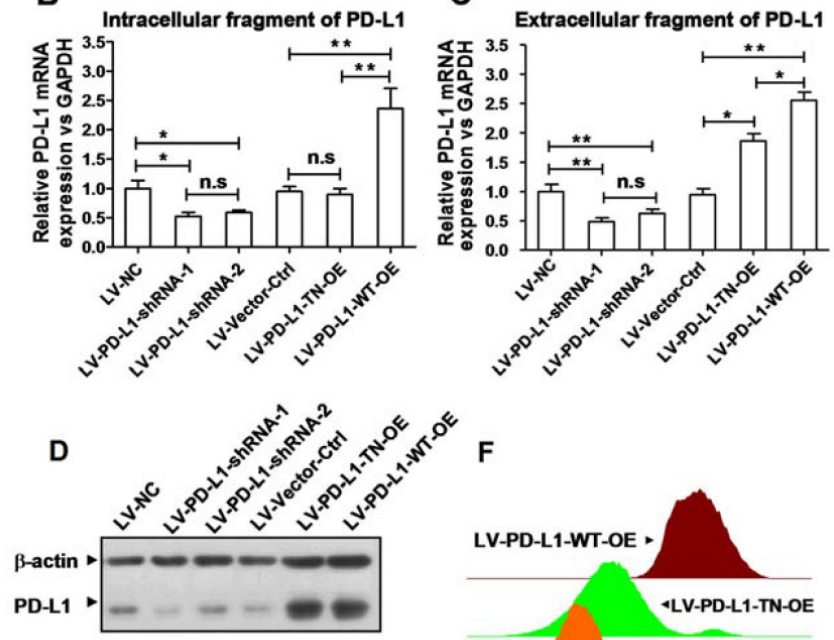

E

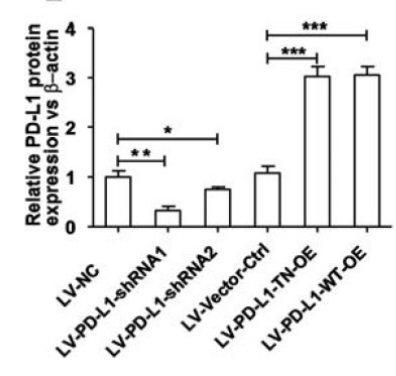

$F$

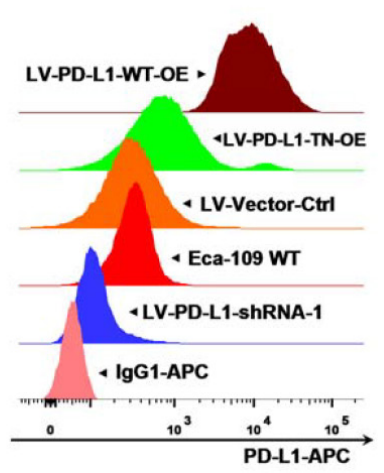

LV-PD-L1-WT-OE cells, but importantly did not change in LV-PD-L1-TN-OE in contrast to LVVector-Ctrl cells. In addition, the mRNA expression level of the extra-cellular fragment of PD-L1 was also reduced in both LV-PD-L1-shRNA-1 and LV-PD-L1-shRNA-2 cells in contrast to LC-NC cells, while it was increased in both LV-PD-L1-WT-OE cells and LV-PD-L1-TN-OE in contrast to LV-Vector-Ctrl cells. GAPDH mRNA expression was used as control. We also confirmed the mRNA expression of both the intracellular and extra-cellular fragments of PD-L1 in different cellular models using a real-time PCR assay. As seen in Fig. 3B and C, we observed a similar mRNA expression pattern to the one explained above. Finally, we also checked the PD-L1 protein expression in these different cellular models by western blot. As shown in Fig. 3D, PD-L1 ablation by shRNA 1 and 2 (lanes 2 and 3) significantly reduced its expression as compared to the vector control (lane 1). Similarly, over-expression of wild type and cytoplasmic truncated PD-L1 showed significant up-regulation in comparison to LV-Vector-Ctrl. Fig. 3E shows the significant differences in the normalized protein expression of PD-L1 normalized to $\beta$-actin protein in different cellular models of Eca-109 cells. We also confirmed the surface expression of PD-L1 in these different cell types by flow cytometry (Fig. 3F).

\section{KARGER}




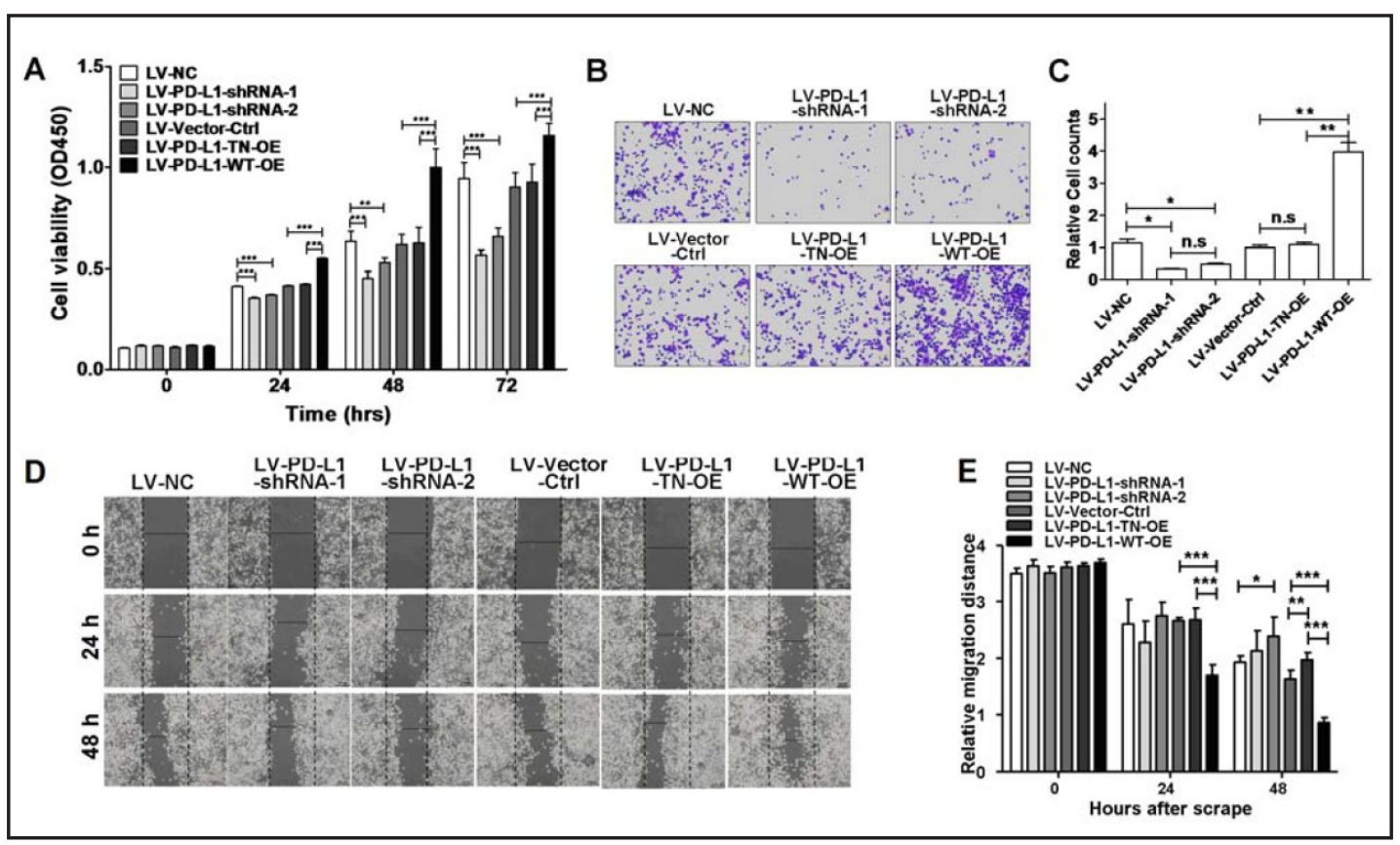

Fig. 4. Effects of PD-L1 expression on cell viability and migration of Eca-109 cells. A. Cell viability analysis of different cellular models of Eca-109 cells 24, 48 and 72 hrs after plating, and the quantitation of cell viability from multiple experiments was measured. B. Cell migration analysis of different cellular models of Eca-109 cells, expressing varying levels of PD-L1 by transwell assay. Pictures represent the number of cells that migrated through matrigel stained with crystal violet dye. C. Quantification of the relative numbers of cells in transwell assay that migrated through matrigel from each cellular model in multiple experiments. D. Cell migration analysis of Eca109 different cellular models to assess the role of PD-L1 by wound healing assay. E. Panel E shows the relative migration distance in a bar graph which evaluated the quantitation of migration distances from multiple experiments. "*” represents a $P$ value of $<0.05$, while "**" represents a $P$ value of $<0.01$, and “***” represents a $P$ value of $<0.001$.

Fig. 5. Analysis of the effects of PD-L1 expression on mRNA levels of EMT markers. RT-PCR based mRNA expression analysis of various EMT marker genes in the different Eca-109 cellular models. The relative expression data has been normalized against GAPDH mRNA expression. “*” represents a $P$ value of $<0.05$, while “**” represents a $P$ value of $<0.01$, and “***" represents a $P$ value of $<0.001$.

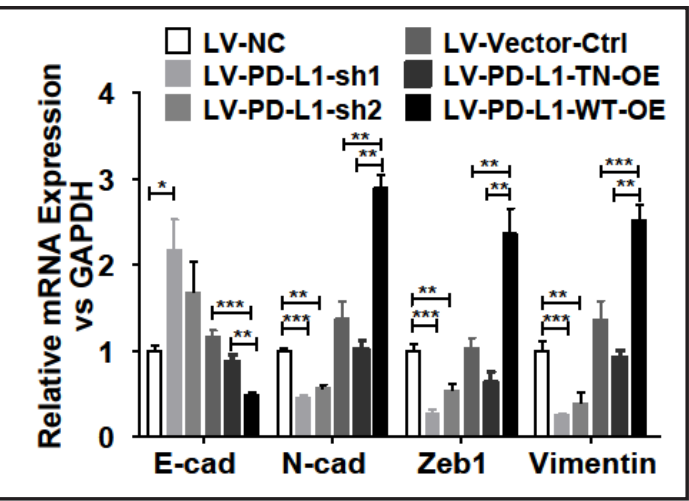

PD-L1 expression promoted Eca-109 cell viability and migration

Analysis of the contribution of PD-L1 in regulating the in vitro cell viability of ECa109 cells by CCK-8 assay revealed that PD-L1 over-expressing (LV-PD-L1-WT-OE) cells had significantly higher cell viability than LV-PD-L1-TN-OE or LV-Vector-Ctrl group cells at 24, 48 and 72 hour time points. Similarly, PD-L1 ablation (LV-PD-L1-shRNA-1 or LV-PD-L1shRNA-2) significantly reduced cell viability at 24, 48 and 72 hour time points in comparison to cells from LV-NC group. This is shown in Fig. 4A.

We also used the transwell assay to examine the contribution of PD-L1 expression in regulating migration ability of Eca-109 cells. As shown in Fig. 4B and C, the number of crystal violet stained migrated cells were significantly less in LV-PD-L1-shRNA-1 and LV-PD- 
A

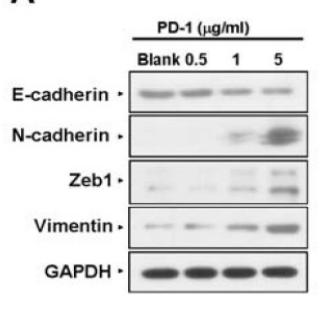

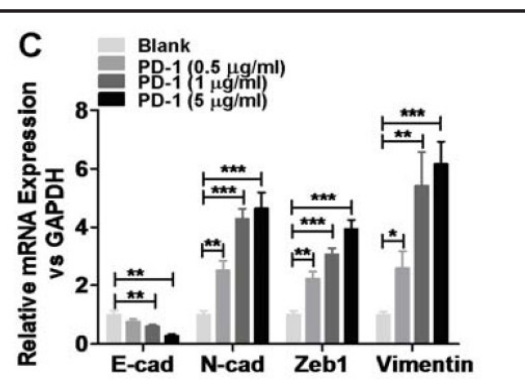

Fig. 6. PD-1 fusion protein treatment promotes EMT phenotype in PD-L1 over-expressing Eca-109 cells. Panel A represents the protein expression of different EMT markers (E-cadherin, N-cadherin, Zeb-1 and Vimentin) in Eca-109 cells over-expressing PD-L1 (LV-PD-L1-WT-OE) after treatment with different concentrations $(0.5,1,5 \mu \mathrm{g} / \mathrm{ml})$ of PD-1 fusion protein. Panel B shows the relative protein expression of these markers after normalization with GAPDH protein from multiple experiments. Panel C shows the relative mRNA expression of EMT marker genes in cells over-expressing PD-L1 after treatment with PD-1 fusion protein, and normalized with GAPDH expression. "*" represents a $P$ value of $<0.05$, while "**" represents a $P$ value of $<0.01$, and “***” represents a $P$ value of $<0.001$.

L1-shRNA-2 groups in contrast to LV-NC group ( $P<0.05$ respectively). Consistently, PD-L1 overexpression (LV-PD-L1-WT-OE group) significantly increased the number of migrated cells compared to cells from LV-PD-L1-TN-OE and LV-PD-L1-Vector-Ctrl groups.

Similarly, we also analyzed the role of PD-L1 in impacting the migration ability of Eca109 cells by performing the wound healing assay. As seen in Fig. 4 D and E, 24 and 48 hrs after inducing the scratch, the cell-free area in cells over-expressing PD-L1 (LV-PD-L1-WTOE) was significantly narrower than that of LV-PD-L1-TN-OE and LV-Vector-Ctrl groups, respectively. Consistent with this finding, cells with PD-L1 ablation (LV-PD-L1-shRNA-2) showed a significantly wider gap after scratch administration in comparison to control ablation (LV-Vector-Ctrl) group cells.

\section{PD-L1 expression promoted EMT phenotype in Eca-109 cells}

Next, we investigated the effect of PD-L1 on the EMT phenotype in Eca-109 cells by analyzing the expression of various EMT markers including E-cadherin, $\mathrm{N}$-cadherin, Zeb1 and vimentin. Our RT-PCR based mRNA expression analysis of these genes (Fig. 5) revealed that PD-L1 ablation by both shRNAs (LV-PD-L1-shRNA-1 or LV-PD-L1-shRNA-2) increased E-cadherin expression (but only shRNA1 showed significant change), while its overexpression (LV-PD-L1- WT-OE) significantly reduced the expression, in contrast to LV-Vector-Ctrl and LV-PD-L1-TN-OE group Eca-109 cells. However, mRNA expression levels of N-cadherin, Zeb1 and vimentin were significantly decreased when PD-L1 was ablated with either of the shRNA (LV-PD-L1-shRNA-1 or LV-PD-L1-shRNA-2), in contrast to LV-NC, while PD-L1 overexpression (LV-PD-L1- WT-OE) significantly increased their expression in contrast to LV-PD-L1-TN-OE and LV-NC group Eca-109 cells. Overall, our data demonstrated that PD-L1 overexpression enhanced the mesenchymal markers (N-cadherin, Zeb1 and vimentin) and thus contributed to the EMT phenotype of Eca-109 cells.

\section{Binding of exogenous $P D-1$ receptor fusion protein to PD-L1 promoted EMT phenotype}

Herein, we tested if PD-1 receptor binding with PD-L1 has any effect on the PD-L1 mediated promotion of EMT in Eca-109 cells. We stimulated PD-L1 over-expressing Eca-109 (LV-PD-L1-WT-OE) cells with different concentrations of PD-1 fusion protein (recombinant human PD-1 Fc chimera) for 24 hrs, and as seen in Fig. 6A and B, cells treated with 1 and 5 $\mu \mathrm{g} / \mathrm{ml}$ of PD-1 fusion protein showed significantly reduced E-cadherin protein expression in comparison to the no treatment group (Blank) or low concentration $(0.5 \mu \mathrm{g} / \mathrm{ml})$. However, the expression levels of $\mathrm{N}$-cadherin and Zeb1 proteins were significantly increased upon PD-1 stimulation under both concentrations ( 1 and $5 \mu \mathrm{g} / \mathrm{ml}$ ). Notably, the vimentin protein expression was significantly increased by all three different concentrations of PD-1 fusion 
Fig. 7. Analysis of the effects of PD-1 fusion protein treatment on EMT phenotype using different cellular models of Eca-109 cells. The different cellular models of Eca-109 cells expressing varying levels of PD-L1 were treated with IgG and PD-1 fusion protein $(5 \mu \mathrm{g} / \mathrm{ml})$ separately for $24 \mathrm{hrs}$. A. This panel represents the expression of different EMT marker proteins (E-cadherin, N-cadherin, Zeb-1 and Vimentin) and GAPDH, as assessed by western blot. B., C., D. \& E., These panels show the relative protein expression of individual EMT marker proteins after normalization with GAPDH protein. “*” represents a $P$ value of $<0.05$, while “**” represents a $P$ value of $<0.01$, and “***” represents a $P$ value of $<0.001$.

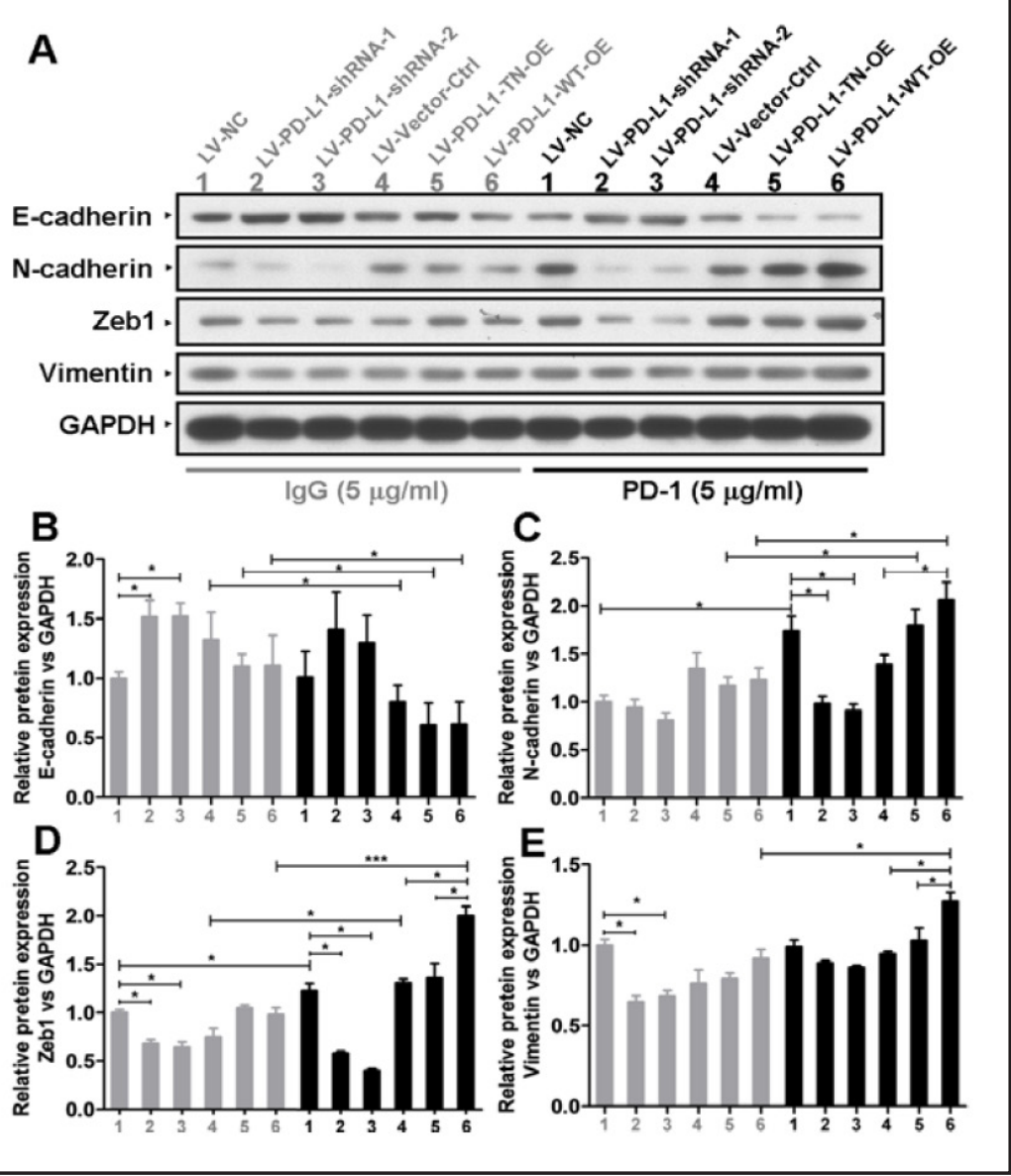

protein. Furthermore, changes in the mRNA expression levels of E-cadherin, N-cadherin, Zeb-1 and Vimentin genes in PD-L1 over-expressing (LV-PD-L1-WT-OE) Eca-109 cells treated with different concentrations of PD-1 fusion protein for $24 \mathrm{hrs}$ were analyzed by RT-PCR. As seen in Fig. 6C, the mRNA expression patterns were consistent with expected changes as observed above in Fig. 6A and B.

We further extended our study to confirm the effects of PD-1 stimulation on PDL1 mediated EMT promotion by using Eca-109 cells, either ablated with PD-L1 or overexpressing cytoplasmic truncated mutant. All cellular models were stimulated with $5 \mu \mathrm{g} / \mathrm{ml}$ of either PD-1 fusion protein or IgG for $24 \mathrm{hrs}$, and the expression of different EMT markers was analyzed by western blot (Fig. 7A). The expression levels of individual proteins were later normalized to GAPDH expression. As shown in Fig. 7B, the protein levels of E-cadherin in cells from LV-Vector-Ctrl, LV-PD-L1-TN-OE and LV-PD-L1-WT-OE groups were significantly reduced after PD-1 treatment (black bars, lanes 4, 5 \& 6), in contrast to corresponding IgG treatment (grey bars, lanes 4, 5 \& 6). But PD-1 treatment in cells with PD-L1 ablation (black bars, lane $2 \& 3$ ) did not show any effect, suggesting that effects are PD-L1 specific. However, the protein levels of N-cadherin and Zeb1 in LV-NC, LV-PD-L1-TN-OE and LV-PD-L1-WT-OE group cells significantly increased after PD-1 treatment in comparison to their IgG treated counterparts, as shown in Fig. 7C, while the effects were not evident in cells with PD-L1 ablation. Similar significant differences were observed in the expression patterns of Zeb1 and vimentin proteins after PD-1 stimulation in different cell types as compared to IgG stimulation (Fig. 7 D \& E). 


\section{Discussion}

Many recent studies have highlighted the critical role of PD-L1 in regulating the biological functions of cancer cells themselves, including cell proliferation, migration, metastasis, chemo/radio-resistance, and the promotion of epithelial to mesenchymal transition $[9,10,18,21,23,30-32]$. It has been suggested that signal pathways such as JAK/ STAT1/IRF-1, MEK/ERK/STAT1 and PKD2 etc. were involved in the regulation of PD-L1 expression, and these pathways could initiate transcription factors including IRF-1, STAT1 or STAT3, which target the promoter of PD-L1 [33-35]. Meanwhile, the abnormal expression of PD-L1 in cancer cells could induce a series of cellular responses that are associated with the prevention of apoptosis, failure of chemo/radio treatment and immune clearance. We have also previously demonstrated that the level of PD-L1 expression could be used as an important prognostic predictor for esophageal cancer patients and its increased expression was significantly associated with increased tumor migration [24]. Moreover, in a separate study we also reported on the significant association between EMT status of human esophageal cancer patients and tumor migration, metastasis and postoperative prognosis [25]. But, the mechanistic link between the abnormal expression of PD-L1 in human esophageal cancer tissues and its contribution to the EMT phenotype still remains elusive. In the present study, we have investigated this correlation and the contribution of PD-L1 in regulating EMT using human esophageal cancer cell line Eca-109.

EMT is one of the critical steps in the early stages of cancer metastasis. During the physio-pathological process of EMT, epithelial cells interact with surrounding mesenchymal cells, and obtain certain mesenchymal phenotypes, including changes in morphology, polarity, surface markers, and related transcription factors [36-41]. Once the cancer cells obtain mesenchymal phenotypes, they gain the ability to migrate into the stroma or migrate to distant sites and organs, and finally contribute to tumor metastasis and recurrence [42, 43]. In addition, numerous studies have also indicated that cancer cells undergoing EMT attain certain characteristics of cancer stem cells, which leads to chemo/radio resistance and tumor immune escape [44]. It is noteworthy that PD-L1 has been considered as an important mediator of EMT in certain human cancer tissues, such as lung cancer, colorectal cancer, and head and neck cancer [21]. In our present study, we have also confirmed this relationship between PD-L1 expression and EMT status in human esophageal cancer tissues, and this led us to probe deeper into the mechanism of PD-L1 mediated regulation of EMT in esophageal cancer cells.

To delineate this mechanistic contribution, we first established multiple cellular models using the Eca-109 esophageal cancer cell line, either by ablating PD-L1 or over-expressing its wild type or cytoplasmic truncated mutant. Using these different cellular models, we have been able to establish that PD-L1 significantly promoted cancer cell viability and migration. We also demonstrated that in Eca-109 cells, PD-L1 overexpression altered the expression of many EMT marker genes like E-cadherin, N-cadherin, Zeb-1 and Vimentin, which was consistent with EMT promotion. Thus, our data supported the hypothesis that PD-L1 is indeed involved in the malignant transformation of esophageal cancer cells and plays an important role in driving EMT.

To further uncover additional mechanistic details of the role of PD-L1 in influencing cellular functions of esophageal cancer cells, we used its cytoplasmic domain mutant. The cytoplasmic domain mutant has previously been shown to be involved in transferring a reverse signal to tumor cells from the PD-1 receptor on immune cells [45]. Our study confirmed that overexpression of this PD-L1 mutant on Eca-109 did not influence any of their cellular functions. Moreover, change in EMT markers (E-cadherin, N-cadherin, Zeb1 and vimentin) was not observed upon PD-1 stimulation when the cytoplasmic domain mutant of PD-L1 was expressed. These experiments highlighted the importance of the cytoplasmic domain of PD-L1 in inducing signals to regulate various cellular functions. It has been demonstrated that the positive relationship between Zeb1 and PD-L1 expression in human esophageal cancer tissues, and the gene promoter region of PD-L1 also contains a 


\section{Cellular Physiology Cell Physiol Biochem 2017;42:2267-2280 \begin{tabular}{l|l|l} 
DOI: 10.1159/000480000 & and Biochemistry Published online: August 17, 2017 & $\begin{array}{l}\text { O 2017 The Author(s). Published by S. Karger AG, Basel } \\
\text { www.karger.com/cpb }\end{array}$
\end{tabular}}

binding site for ZEB1 [46]. Mechanistic investigations revealed that siRNA silencing of Zeb-1 and overexpression of miR200 family members in MCF7 sh-WISP2 cells strongly decreased PD-L1 expression, which also suggested that the transcription factor Zeb1 was involved in the PD-L1 mediated EMT activation [47].

Overall, our present study explored the cross-talk between PD-L1 expression and EMT during cancer progression, suggested that PD-L1 is very important in inducing the EMT phenotype in esophageal cancer cells, and showed that this induction is mediated by the cytoplasmic domain of PD-L1.

\section{Acknowledgments}

This work was supported by grants from the National Natural Science Foundation of China (No. 81301960, 31428005, 31570877 and 31570908), the Key R\&D Project of Science and Technology Department of Jiangsu Province (BE2015633, BE2015634 and BE2016660), Changzhou High-Level Medical Talents Training Project (No. 2016CZBJ001 and 2016CZLJ022). This work was also supported in part by R21CA205727, a Head and Neck Cancer Spore Development award, a pilot grant of Roswell Park Cancer Institute/University of Pittsburgh Cancer Institute Ovarian Cancer Specialized Programs of Research Excellence Grants P50CA159981, and the AAI (American Association of Immunologists) Careers in Immunology Fellowship Program.

\section{Disclosure Statement}

The authors declare no conflicts of interest.

\section{References}

1 Dong H, Zhu G, Tamada K, Chen L: B7-H1, a third member of the B7 family, co-stimulates T-cell proliferation and interleukin-10 secretion. Nat Med 1999;5:1365-1369.

2 Ceeraz S, Nowak EC, Noelle RJ: B7 family checkpoint regulators in immune regulation and disease. Trends Immunol 2013;34:556-563.

3 Wang Y, Wu J, Guo W, Sun Q, Chen X, Zang W, Dong Z, Zhao G: alpha-Solanine Modulates the Radiosensitivity of Esophageal Cancer Cells by Inducing MicroRNA 138 Expression. Cell Physiol Biochem 2016;39:9961010.

4 Li Z, Dong P, Ren M, Song Y, Qian X, Yang Y, Li S, Zhang X, Liu F: PD-L1 Expression Is Associated with Tumor FOXP3(+) Regulatory T-Cell Infiltration of Breast Cancer and Poor Prognosis of Patient. J Cancer 2016;7:784-793.

5 Chen K, Cheng G, Zhang F, Zhang N, Li D, Jin J, Wu J, Damaraju S, Mao W, Su D: Prognostic significance of programmed death-1 and programmed death-ligand 1 expression in patients with esophageal squamous cell carcinoma. Oncotarget 2016;10.18632/oncotarget.8956

-6 Brahmer JR, Tykodi SS, Chow LQ, Hwu WJ, Topalian SL, Hwu P, Drake CG, Camacho LH, Kauh J, Odunsi K, Pitot HC, Hamid O, Bhatia S, Martins R, Eaton K, Chen S, Salay TM, Alaparthy S, Grosso JF, Korman AJ, Parker SM, Agrawal S, Goldberg SM, Pardoll DM, Gupta A, Wigginton JM: Safety and activity of anti-PD-L1 antibody in patients with advanced cancer. N Engl J Med 2012;366:2455-2465.

7 Bagley SJ, Bauml JM, Langer CJ: PD-1/PD-L1 immune checkpoint blockade in non-small cell lung cancer. Clin Adv Hematol Oncol 2015;13:676-683.

-8 Lee Y, Shin JH, Longmire M, Wang H, Kohrt HE, Chang HY, Sunwoo JB: CD44+ cells in head and neck squamous cell carcinoma suppress T cell-mediated immunity by selective constitutive and inducible expression of PD-L1. Clin Cancer Res 2016;10.1158/1078-0432.CCR-15-2665

-9 Lou Y, Diao L, Parra Cuentas ER, Denning WL, Chen L, Fan YH, Byers LA, Wang J, Papadimitrakopoulou VA, Behrens C, Rodriguez J, Hwu P, Wistuba, II, Heymach JV, Gibbons DL: Epithelial-mesenchymal transition 


\section{Cellular Physiology Cell Physiol Biochem 2017;42:2267-2280 \begin{tabular}{ll|l} 
and Biochemistry Published onlıne: August 17, 2017 & $\begin{array}{l}\text { (c) } 2017 \text { The Author(s). Published by S. Karger AG, Basel } \\
\text { www.karger.com/cpb }\end{array}$
\end{tabular} \\ Chen et al.: PD-L1 Promotes EMT in Esophageal Cancer}

is associated with a distinct tumor microenvironment including elevation of inflammatory signals and multiple immune checkpoints in lung adenocarcinoma. Clin Cancer Res 2016;10.1158/1078-0432.CCR-151434

10 Alsuliman A, Colak D, Al-Harazi O, Fitwi H, Tulbah A, Al-Tweigeri T, Al-Alwan M, Ghebeh H: Bidirectional crosstalk between PD-L1 expression and epithelial to mesenchymal transition: significance in claudin-low breast cancer cells. Mol Cancer 2015;14:149.

11 Dezutter-Dambuyant C, Durand I, Alberti L, Bendriss-Vermare N, Valladeau-Guilemond J, Duc A, Magron A, Morel AP, Sisirak V, Rodriguez C, Cox D, Olive D, Caux C: A novel regulation of PD-1 ligands on mesenchymal stromal cells through MMP-mediated proteolytic cleavage. Oncoimmunology 2016;5:e1091146.

-12 Schehr JL, Schultz ZD, Warrick JW, Guckenberger DJ, Pezzi HM, Sperger JM, Heninger E, Saeed A, Leal T, Mattox K, Traynor AM, Campbell TC, Berry SM, Beebe DJ, Lang JM: High Specificity in Circulating Tumor Cell Identification Is Required for Accurate Evaluation of Programmed Death-Ligand 1. PLoS One 2016;11:e0159397.

13 Chen S, Wang RX, Liu Y, Yang WT, Shao ZM: PD-L1 expression of the residual tumor serves as a prognostic marker in local advanced breast cancer after neoadjuvant chemotherapy. Int J Cancer 2016;10.1002/ ijc.30552

14 Mesnage SJ, Auguste A, Genestie C, Dunant A, Pain E, Drusch F, Gouy S, Morice P, Bentivegna E, Lhomme C, Pautier P, Michels J, Le Formal A, Cheaib B, Adam J, Leary AF: Neoadjuvant chemotherapy (NACT) increases immune infiltration and programmed death-ligand 1 (PD-L1) expression in epithelial ovarian cancer (EOC). Ann Oncol 2016;10.1093/annonc/mdw625

15 Li J, Chen L, Xiong Y, Zheng X, Xie Q, Zhou Q Shi L, Wu C, Jiang J, Wang H: Knockdown of PD-L1 in Human Gastric Cancer Cells Inhibits Tumor Progression and Improves the Cytotoxic Sensitivity to CIK Therapy. Cell Physiol Biochem 2017;41:907-920.

-16 Shi SJ, Wang LJ, Wang GD, Guo ZY, Wei M, Meng YL, Yang AG, Wen WH: B7-H1 expression is associated with poor prognosis in colorectal carcinoma and regulates the proliferation and invasion of HCT116 colorectal cancer cells. PLoS One 2013;8:e76012.

17 Cao Y, Zhang L, Kamimura Y, Ritprajak P, Hashiguchi M, Hirose S, Azuma M: B7-H1 overexpression regulates epithelial-mesenchymal transition and accelerates carcinogenesis in skin. Cancer Res 2011;71:1235-1243.

18 Cao Y, Zhang L, Ritprajak P, Tsushima F, Youngnak-Piboonratanakit P, Kamimura Y, Hashiguchi M, Azuma M: Immunoregulatory molecule B7-H1 (CD274) contributes to skin carcinogenesis. Cancer Res 2011;71:4737-4741.

19 Inaguma S, Lasota J, Wang Z, Felisiak-Golabek A, Ikeda H, Miettinen M: Clinicopathologic profile, immunophenotype, and genotype of CD274 (PD-L1)-positive colorectal carcinomas. Mod Pathol 2016;10.1038/modpathol.2016.185

20 Zhi Y, Mou Z, Chen J, He Y, Dong H, Fu X, Wu Y: B7H1 Expression and Epithelial-To-Mesenchymal Transition Phenotypes on Colorectal Cancer Stem-Like Cells. PLoS One 2015;10:e0135528.

21 Kim S, Koh J, Kim MY, Kwon D, Go H, Kim YA, Jeon YK, Chung DH: PD-L1 expression is associated with epithelial-to-mesenchymal transition in adenocarcinoma of the lung. Hum Pathol 2016;58:7-14.

-22 Wang Y, Wang H, Zhao Q Xia Y, Hu X, Guo J: PD-L1 induces epithelial-to-mesenchymal transition via activating SREBP-1c in renal cell carcinoma. Med Oncol 2015;32:212.

-23 Ock CY, Kim S, Keam B, Kim M, Kim TM, Kim JH, Jeon YK, Lee JS, Kwon SK, Hah JH, Kwon TK, Kim DW, Wu HG, Sung MW, Heo DS: PD-L1 expression is associated with epithelial-mesenchymal transition in head and neck squamous cell carcinoma. Oncotarget 2016;7:15901-15914.

-24 Chen L, Deng H, Lu M, Xu B, Wang Q Jiang J, Wu C: B7-H1 expression associates with tumor invasion and predicts patient's survival in human esophageal cancer. Int J Clin Exp Pathol 2014;7:6015-6023.

-25 Liu J, Chen L, Deng H, Xu B, Li M, Zheng X, Wu C, Jiang J: Epithelial-to-mesenchymal transition in human esophageal cancer associates with tumor progression and patient's survival. Int J Clin Exp Pathol 2014;7:6943-6949.

26 Chen L, Di D, Luo G, Zheng L, Tan Y, Zhang X, Xu N: Immunochemical staining of MT2-MMP correlates positively to angiogenesis of human esophageal cancer. Anticancer Res 2010;30:4363-4368.

27 Chen L, Sun J, Wu H, Zhou S, Tan Y, Tan M, Shan B, Lu B, Zhang X: B7-H4 expression associates with cancer progression and predicts patient's survival in human esophageal squamous cell carcinoma. Cancer Immunol Immunother 2011;60:1047-1055. 


\section{Cellular Physiology Cell Physiol Biochem 2017;42:2267-2280 \begin{tabular}{ll|l} 
and Biochemistry Published onlıne: August 17, 2017 & $\begin{array}{l}\text { (c) } 2017 \text { The Author(s). Published by S. Karger AG, Basel } \\
\text { www.karger.com/cpb }\end{array}$
\end{tabular}}

Chen et al.: PD-L1 Promotes EMT in Esophageal Cancer

28 Chen L, Chen J, Xu B, Wang Q, Zhou W, Zhang G, Sun J, Shi L, Pei H, Wu C, Jiang J: B7-H3 expression associates with tumor invasion and patient's poor survival in human esophageal cancer. Am J Transl Res 2015; 7:2646-2660.

-29 Zhou Q Zheng X, Chen L, Xu B, Yang X, Jiang J, Wu C: Smad2/3/4 Pathway Contributes to TGF-beta-Induced MiRNA-181b Expression to Promote Gastric Cancer Metastasis by Targeting Timp3. Cell Physiol Biochem 2016;39:453-466.

-30 Cao Y, Zhang L, Kamimura Y, Ritprajak P, Hashiguchi M, Hirose S, Azuma M: B7-H1 overexpression regulates epithelial-mesenchymal transition and accelerates carcinogenesis in skin. Cancer Res 2010;71:1235-1243.

-31 Hirai M, Kitahara H, Kobayashi Y, Kato K, Bou-Gharios G, Nakamura H, Kawashiri S: Regulation of PD-L1 expression in a high-grade invasive human oral squamous cell carcinoma microenvironment. Int J Oncol 2017;50:41-48.

-32 Satelli A, Batth IS, Brownlee Z, Rojas C, Meng QH, Kopetz S, Li S: Potential role of nuclear PD-L1 expression in cell-surface vimentin positive circulating tumor cells as a prognostic marker in cancer patients. Sci Rep 2016;6:28910.

-33 Concha-Benavente F, Srivastava RM, Trivedi S, Lei Y, Chandran U, Seethala RR, Freeman GJ, Ferris RL: Identification of the cell-intrinsic and extrinsic pathways downstream of EGFR and IFNgamma that induce PD-L1 expression in head and neck cancer. Cancer Res 2015;10.1158/0008-5472.CAN-15-2001

-34 Cortez MA, Ivan C, Valdecanas D, Wang X, Peltier HJ, Ye Y, Araujo L, Carbone DP, Shilo K, Giri DK, Kelnar K, Martin D, Komaki R, Gomez DR, Krishnan S, Calin GA, Bader AG, Welsh JW: PDL1 Regulation by p53 via miR-34. J Natl Cancer Inst 2016;108:

-35 Peng D, Kryczek I, Nagarsheth N, Zhao L, Wei S, Wang W, Sun Y, Zhao E, Vatan L, Szeliga W, Kotarski J, Tarkowski R, Dou Y, Cho K, Hensley-Alford S, Munkarah A, Liu R, Zou W: Epigenetic silencing of TH1-type chemokines shapes tumour immunity and immunotherapy. Nature 2015;527:249-253.

-36 Acloque H, Adams MS, Fishwick K, Bronner-Fraser M, Nieto MA: Epithelial-mesenchymal transitions: the importance of changing cell state in development and disease. J Clin Invest 2009;119:1438-1449.

37 Zeisberg M, Neilson EG: Biomarkers for epithelial-mesenchymal transitions. J Clin Invest 2009;119:14291437.

38 Kalluri R, Weinberg RA: The basics of epithelial-mesenchymal transition. J Clin Invest 2009;119:14201428.

39 Liu B, Jia Y, Cao Y, Wu S, Jiang H, Sun X, Ma J, Yin X, Mao A, Shang M: Overexpression of Phosphoserine Aminotransferase 1 (PSAT1) Predicts Poor Prognosis and Associates with Tumor Progression in Human Esophageal Squamous Cell Carcinoma. Cell Physiol Biochem 2016;39:395-406.

40 Yan S, Jiang H, Fang S, Yin F, Wang Z, Jia Y, Sun X, Wu S, Jiang T, Mao A: MicroRNA-340 Inhibits Esophageal Cancer Cell Growth and Invasion by Targeting Phosphoserine Aminotransferase 1. Cell Physiol Biochem 2015;37:375-386.

-41 Niu H, Gong L, Tian X, Fang L, Wang C, Zhu Y: Low Expression of miR-491 Promotes Esophageal Cancer Cell Invasion by Targeting TPX2. Cell Physiol Biochem 2015;36:2263-2273.

42 Ye X, Weinberg RA: Epithelial-Mesenchymal Plasticity: A Central Regulator of Cancer Progression. Trends Cell Biol 2015;25:675-686.

43 Liu P, Gao Y, Huan J, Ge X, Tang Y, Shen W, Tian Y, Shen W, Zou S, Zhou J, Zhang S: Upregulation of PAX2 promotes the metastasis of esophageal cancer through interleukin-5. Cell Physiol Biochem 2015;35:740754.

44 Yao C, Su L, Shan J, Zhu C, Liu L, Liu C, Xu Y, Yang Z, Bian X, Shao J, Li J, Lai M, Shen J, Qian C: IGF/STAT3/ NANOG/Slug signaling axis simultaneously controls epithelial-mesenchymal transition and stemness maintenance in colorectal cancer. Stem Cells 2016;10.1002/stem.2320

45 Azuma T, Yao S, Zhu G, Flies AS, Flies SJ, Chen L: B7-H1 is a ubiquitous antiapoptotic receptor on cancer cells. Blood 2008;111:3635-3643.

46 Tsutsumi S, Saeki H, Nakashima Y, Ito S, Oki E, Morita M, Oda Y, Okano S, Maehara Y: PD-L1 expression at tumor invasive front is associated with EMT and poor prognosis in esophageal squamous cell carcinoma. Cancer Sci 2017;10.1111/cas.13237

47 Noman MZ, Janji B, Abdou A, Hasmim M, Terry S, Tan TZ, Mami-Chouaib F, Thiery JP, Chouaib S: The immune checkpoint ligand PD-L1 is upregulated in EMT-activated human breast cancer cells by a mechanism involving ZEB-1 and miR-200. Oncoimmunology 2017;6:e1263412. 